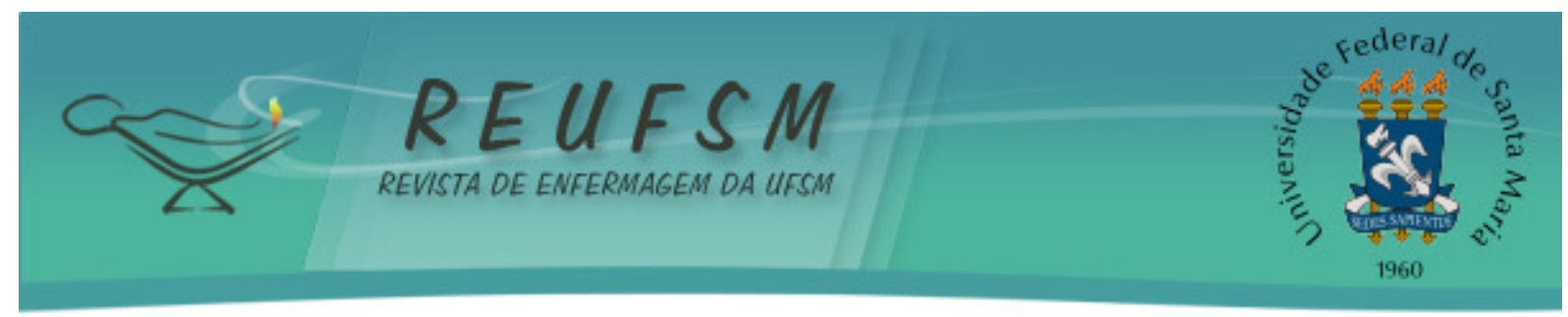

ARTIGO ORIGINAL

\title{
ÍNDICE DE TREINAMENTO DE ENFERMAGEM ENQUANTO INDICADOR DE QUALIDADE DE GESTÃO DE RECURSOS HUMANOS
}

\author{
INDEX OF NURSING TRAINING AS INDICATOR OF QUALITY OF HUMAN RESOURCE \\ MANAGEMENT
}

ÍNDICE DE ENTRENAMIENTO DE ENFERMERÍA

COMO INDICATIVO DE CALIDAD DE GESTIÓN DE RECURSOS HUMANOS

\author{
João Lucas Campos de Oliveira ${ }^{1}$ \\ Anair Lazzari Nicola ${ }^{2}$ \\ Doi: $10.5902 / 217976928772$ \\ Angela Elisa Breda Rodrigues de Souza ${ }^{3}$
}

RESUMO: Objetivo: determinar o índice de treinamento de profissionais de enfermagem. Método: estudo retrospectivo, descritivo com abordagem quantitativa. Desenvolvido em um hospital de ensino no estado do Paraná com objeto de estudo as atividades de treinamento direcionadas para os profissionais de enfermagem. Resultados: o índice de treinamento encontrado foi de 16,20 para os profissionais enfermeiros e de 3,98 para técnicos e auxiliares de enfermagem. Conclusão: os valores encontrados do índice de treinamento podem ser interpretados como favoráveis aos profissionais enfermeiros e desfavoráveis aos profissionais de enfermagem de nível médio, quando estes comparados a escassa literatura produzida pela enfermagem.

Descritores: Assistência de enfermagem; Recursos humanos; Capacitação em serviço; Gestão de qualidade.

ABSTRACT: aim: determine the index of training of nursing professionals. Method: this paper consists in a retrospective, descriptive study based on a quantitative approach. It was developed in a teaching hospital in Paraná State and it focused on training activities related to nursing professionals as its object of study. Results: the index of training was found is about 16,20 related to nursing professionals and about 3,98 to technical nursing assistants and auxiliaries. Conclusion: the numbers found in this training index can be understood as favorable (positive) concerning about nursing professionals and unfavorable (negative) in relation to a nursing professional of meddium level (position), when all of them are compared to the shortage of literature produced by the nursing.

Descriptors: Nursing care; Human resources; Inservice training; Quality management.

RESUMEN: Objetivo: determinar el índice de entrenamiento de profesionales de enfermería. Método: estudio retrospectivo, descriptivo, con abordaje cuantitativo. Desarrollado en un hospital escuela en el estado del Paraná considerando como objeto de estudio las actividades de entrenamiento direccionadas a los profesionales de enfermería. Resultados: el índice de entrenamiento encontrado fue de 16,20 para los profesionales enfermeros y de 3,98 para profesionales técnicos y auxiliares de enfermería. Conclusión: los valores del índice de entrenamiento pueden ser interpretados como favorables a los

\footnotetext{
${ }^{1}$ Enfermeiro. Mestrando no Programa de Pós-Graduação em Enfermagem (PSE) da Universidade Estadual de Maringá (UEM). Maringá, PR, Brasil. E-mail: enfjoaolcampos@yahoo.com.br

${ }^{2}$ Enfermeira. Doutora em Enfermagem. Docente adjunta do Colegiado de Enfermagem da Universidade Estadual do Oeste do Paraná (UNIOESTE). Coordenadora do Programa de Residência na especialidade de Gerenciamento de Enfermagem em Clínica Médica e Cirúrgica da UNIOESTE. Cascavel, PR, Brasil. E-mail: anairln@yahoo.com.br

${ }^{3}$ Enfermeira. Graduada em Enfermagem pela UNIOESTE. Cascavel, PR, Brasil. E-mail: aebreda@gmail.com
} 


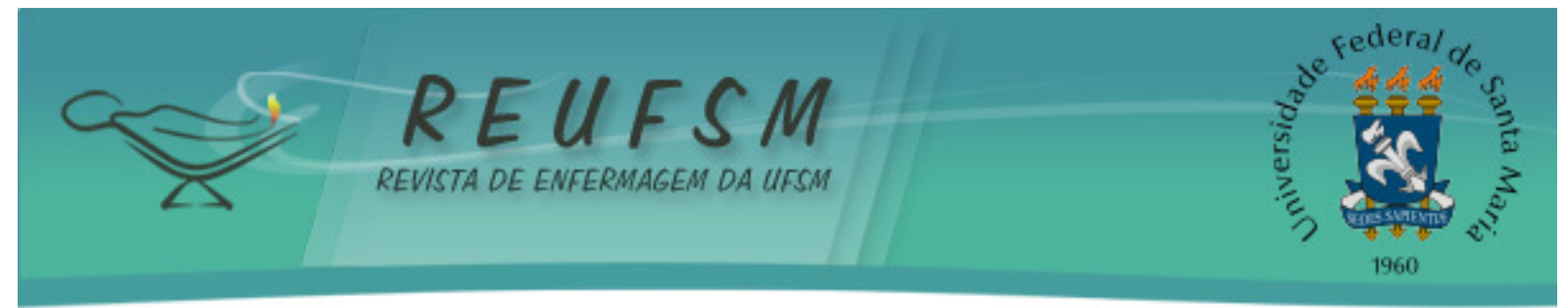

enfermeros profesionales de nivel superior, y desfavorables a los profesionales de enfermería de nivel medio cuando comparados con la escasa literatura producida en el área de Enfermería.

Descriptores: Atencíon de enfermería; Recursos humanos; Capacitación em servicio; Gestión de calidad.

\section{INTRODUÇÃO}

A crescente complexidade do atendimento proporcionado pelos hospitais, considerados as instituições de saúde que mais consomem recursos financeiros e materiais, e o local onde os novos avanços no atendimento e seus riscos associados são mais comumente apresentados, exige que os profissionais atuantes nestes locais mantenham-se atualizados para incorporar os novos conhecimentos e inovações tecnológicas relacionados à assistência.

Os profissionais de enfermagem que prestam assistência em âmbito hospitalar vivenciam diariamente situações que requerem competências para controlar riscos, prever, evitar e minimizar complicações, visando a manutenção da vida e a recuperação do paciente assistido. ${ }^{1}$

Para atender essas demandas, as instituições hospitalares têm adotado novas formas de organização do trabalho visando a qualidade da assistência e a produtividade dos serviços. Para assistir com qualidade, é preciso investimento técnico-científico nos profissionais para que estes estejam aptos a atender melhor e mais seguramente o paciente, assegurar os objetivos da instituição, bem como que reconheçam o investimento proporcionado como forma de satisfação e desenvolvimento no trabalho. ${ }^{2}$

Nesta direção, as habilidades e o desempenho dos trabalhadores de enfermagem devem ser continuamente repensados e melhorados, e para satisfazer a essa exigência necessitam estar envolvidos, principalmente, em atividades de treinamento e avaliação. ${ }^{2}$

0 treinamento é definido como um processo educacional que possibilita às pessoas adquirirem novos conhecimentos, habilidades e atitudes para o desempenho de seus cargos $^{2}$. Pode ser entendido também, como ação sistemática de capacitação e adaptação do indivíduo em uma situação profissional específica, buscando como objetivo o aumento do conhecimento teórico e prático do indivíduo para a realização eficiente de suas atividades profissionais, atividades estas desejáveis no cargo atual ou futuro, ou então habilidades requisitadas pela organização. ${ }^{2}$

No setor saúde e em enfermagem, desenvolver treinamento e qualificação dos recursos humanos vai ao encontro dos princípios da gestão da qualidade. Estas atividades, devem atender de forma integral as necessidades identificadas como deficiências no desempenho do cargo. ${ }^{3}$ Desta forma, compartilhando com os preceitos elencados na administração de recursos humanos, a enfermagem infere que o treinamento dos profissionais deve ser planejado continuamente e de acordo com o modelo assistencial e de gestão dos serviços de enfermagem. ${ }^{3}$

Diante do exposto, menciona-se que o treinamento aos profissionais de enfermagem se faz necessário em várias situações, tais como: necessidade de intervenção pontual na ocorrência de eventos adversos; não conformidade em processos que afetam o bom funcionamento da equipe; resultados dos indicadores assistenciais; necessidade de inovação; atualização e uso adequado de novas tecnologias; a implantação de rotinas; capacitação específica de um procedimento técnico; frequentes episódios de dúvidas de determinados funcionários; aumento de reclamações ou violação repetida de normas técnicas de segurança pelo mesmo funcionário/equipe; entre outros. ${ }^{3-6}$

Outra importante atividade, de cunho gerencial, no tocante aos recursos humanos de enfermagem, é a avaliação da qualidade assistencial. A avaliação deve ser considerada pelos enfermeiros como um processo dinâmico e exaustivo de identificação dos fatores que 


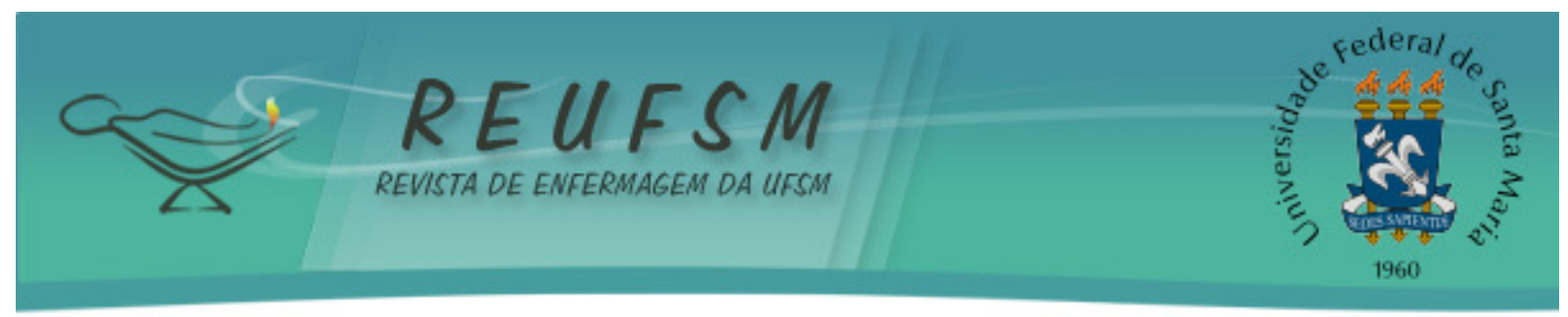

interferem no processo de trabalho da equipe de enfermagem, e requer a adoção de instrumentos que possibilitem avaliar de maneira sistemática os níveis de qualidade dos cuidados prestados. ${ }^{6}$

Uma maneira eficaz de avaliação nos serviços de saúde e de enfermagem é a utilização de indicadores que demonstrem sua evolução ao longo do tempo, permitindo a comparação com referenciais internos e externos. ${ }^{6}$ Destarte, o indicador é uma unidade de medida de uma atividade com a qual está relacionada ou uma medida quantitativa que pode ser usada como um guia para monitorar e avaliar a qualidade assistencial e as atividades de um serviço. ${ }^{6-7}$

No âmbito nacional, o Núcleo de Apoio à Gestão Hospitalar (NAGEH), órgão que compõe o Programa de Compromisso com a Qualidade Hospitalar (CQH), este implementado no estado de São Paulo no início da década de 1990, sistematizou um rol de indicadores relacionados à assistência e ao gerenciamento de recursos humanos de enfermagem, os quais são mensurados periodicamente pelos hospitais que aderiram à metodologia do NAGEH/CQH, com a finalidade de analisar e comparar o desempenho do serviço de enfermagem. ${ }^{7}$ Entre os indicadores de qualidade de gerenciamento de recursos humanos acompanhados pelo NAGEH, encontra-se o Índice de Treinamento de Enfermagem, um indicador de qualidade que busca avaliar a relação entre as horas/homem presentes em atividades de treinamento e as horas/homem trabalhadas. ${ }^{7}$

Cabe aludir, ante ao exposto, que o presente estudo adota a terminologia e conceito treinamento, tendo em vista que esta é utilizada pela área de gestão de recursos humanos ${ }^{2}$, reforçada pelo fato de que, nos serviços de enfermagem, prover ações educativas aos profissionais é uma incumbência privativa do enfermeiro, e que compõe seu trabalho gerencial. ${ }^{5}$ Além disto, a terminologia é adotada na descrição do indicador anteriormente mencionado, objeto de investigação. ${ }^{7}$ Todavia, não se considera como produtivo e salutar a prática unificada de atividades educativas mecanicistas, devendo estas também proporcionar que o trabalhador se insira ativa e reflexivamente na dinâmica organizacional.

Considerando a importância do treinamento dos profissionais de enfermagem para manter a equipe apta a desenvolver assistência segura e de qualidade ao paciente, e da aplicação e análise de indicadores como um meio para avaliar a qualidade dos serviços e dos recursos humanos, optou-se por desenvolver este estudo, utilizando o índice de treinamento da equipe de enfermagem, em sua condição de indicador de qualidade de gerenciamento de recursos humanos.

0 estudo justifica-se, pois o treinamento dos profissionais de enfermagem está intrinsecamente vinculado à qualificação da assistência aos pacientes, algo primordial no exercício do cuidado e da prestação do trabalho em saúde e enfermagem. Nota-se ainda, escassez de estudos na enfermagem perante a temática, pois nas bases de dados como Biblioteca Virtual em Saúde (BVS), Scientific Electronic Library Online (Scielo), Literatura Latino-Americana e do Caribe em Ciências da Saúde (LILACS), PubMed e Base de Dados de Enfermagem (BDEnf) não foram encontradas produções nacionais, com busca realizada no mês de abril de 2013, dos últimos dez anos, específicas ao tema desta pesquisa. Possivelmente, tais estudos podem contribuir para o avanço científico da enfermagem e consequentemente fomentar melhorias adaptadas à prática profissional, principalmente ao que tange às atividades de cunho gerencial do profissional enfermeiro.

Diante da premissa anterior, estabeleceu-se como questão norteadora do estudo: Qual é o índice de treinamento de profissionais de enfermagem em uma organização hospitalar pública e de ensino? E, para responder tal questão, o objetivo consiste em determinar o Índice de Treinamento de Enfermagem de enfermeiros e de profissionais de enfermagem de nível médio, utilizando referencial proposto pelo Núcleo de Apoio à Gestão Hospitalar, sediado no estado de São Paulo. 


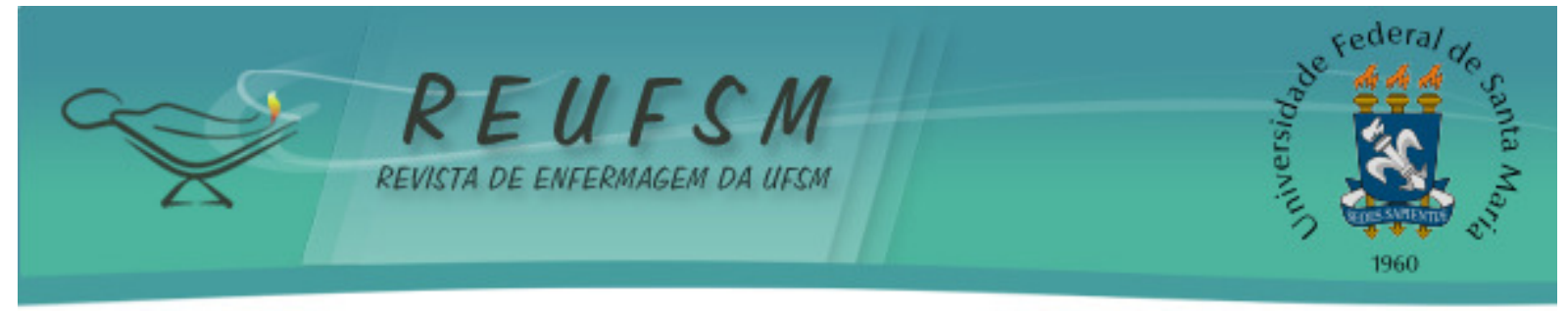

MÉTODO

Trata-se de uma pesquisa retrospectiva, descritiva e exploratória com abordagem quantitativa. Teve como campo de investigação um hospital universitário público situado em Cascavel-PR.

Os dados foram coletados das listas de presença das atividades de treinamento realizadas pelos profissionais da equipe de enfermagem do hospital, em um recorte temporal de abril 2009 a maio de 2010, estabelecido de acordo com a disponibilidade de acesso aos dados e de quando houve o andamento do Projeto que fomentou este e outros estudos acerca de indicadores hospitalares do local em questão. Os dados foram fornecidos pelo setor de Recursos Humanos da mesma organização hospitalar, o qual mantém o registro de todos os treinamentos realizados e é responsável pelo agendamento das atividades, bem como, pelo controle de presença e expedição de comprovante de participação.

Para a coleta de dados, foi elaborada uma planilha para o registro dos dados de cada lista de presença de atividade de treinamento, contendo: data da atividade, tema, carga horária, número de enfermeiros e de técnicos e auxiliares de enfermagem presentes, segregando as categorias. Após a coleta manual dos dados, os mesmos foram transportados para planilha eletrônica para melhor acesso e manejo.

Foram incluídas como atividades de treinamento a totalidade de palestras de curta duração sobre um tema específico, seminários, videoconferências, cursos com temas específicos, e participação em eventos científicos, no recorte temporal determinado e com a participação de pelo menos um profissional de enfermagem, independente de sua categoria. Já como critérios de exclusão delimitaram-se os seguintes: atividades sem informações referentes ao tema do treinamento e/ou carga horária nas fontes de dados consultadas.

Visando a fidedignidade da identificação dos participantes por categoria profissional, utilizou-se uma relação dos nomes de todos os profissionais de enfermagem da instituição, também fornecida pelo setor de Recursos Humanos.

$\mathrm{Na}$ sequência, a análise dos dados ocorreu a partir do cálculo de índice de Treinamento de profissionais de enfermagem proposto pelo $\mathrm{NAGEH}^{7}$, o qual utiliza a fórmula descrita na Figura 1:

$$
I T=\frac{\left(N F_{1} \times C H A T_{1}\right)+\left(N F_{2} \times C H A T_{2}\right)+\ldots+\left(N F_{n} \times C H A T_{n}\right)}{\text { HHT }} \times 1000
$$

IT: indice de treinamento;

NF: número de funcionários participantes do curso;

CHAT: carga horária da atividade de treinamento;

HHT: número de horas homem trabalhadas;

Figura 1: Fórmula do Índice de Treinamento segundo o NAGEH. ${ }^{7}$

A carga horária da atividade de treinamento é a somatória das horas de todas as atividades de treinamento ministradas no período determinado. 0 número de horas/homem trabalhadas é o número de funcionários ativos, do serviço de enfermagem, no cadastro do hospital pelo número de horas previstas para cada um, em contrato de trabalho. ${ }^{7}$

Esta pesquisa foi submetida e aprovada pelo Comitê de Ética em Pesquisa (CEP) da Universidade Estadual do Oeste do Paraná (UNIOESTE), sob o parecer 240/2010 e processo número 598/2010. 


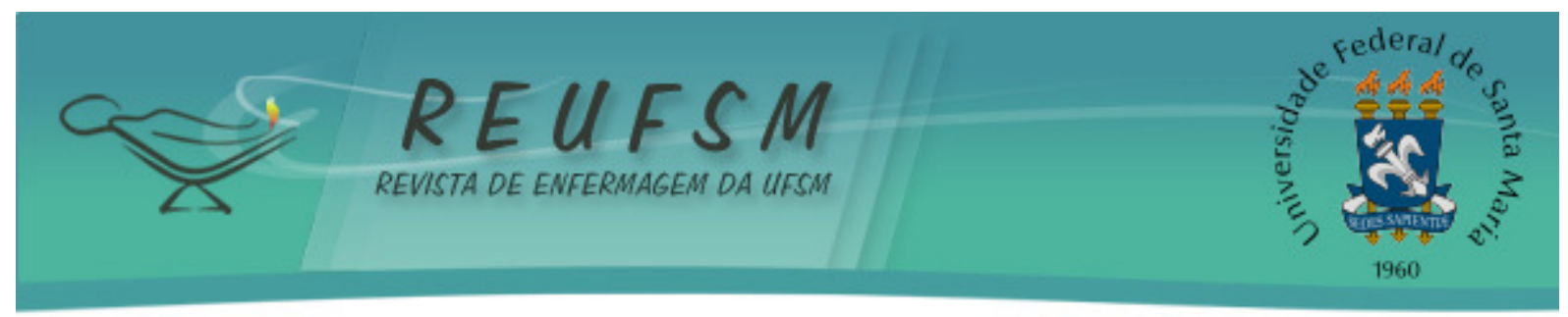

RESULTADOS E DISCUSSÕES

Segundo os dados disponibilizados pelo setor de Recursos Humanos da instituição, a população estudada foi constituída pelos 379 profissionais que compunham o quadro de enfermagem do hospital no recorte temporal estabelecido. Destes, 76 (20,8\%) eram enfermeiros e $303(79,2 \%)$ eram técnicos e/ou auxiliares de enfermagem.

Foram realizadas, no período estudado, 210 atividades de treinamento. Destas, 20 atividades não atenderam a todos os critérios de inclusão, resultando num total de 190 atividades de treinamento analisadas.

Das atividades que conformaram o estudo, os enfermeiros participaram de 155 $(81,6 \%)$, totalizando 335,85 horas totais de treinamento. Os técnicos e auxiliares de enfermagem participaram de 150 atividades (78,9\%) totalizando 293 horas de treinamento. Os profissionais de nível médio foram analisados como uma única categoria, pois no campo onde o estudo foi realizado não existe distinção das atividades exercidas entre elas.

Desta forma, aplicando a fórmula do $\mathrm{NAGEH}^{7}$ encontrou-se o valor de índice de treinamento de 16,20 para profissionais enfermeiros e 3,98 para técnicos e auxiliares de enfermagem.

Poucas foram as produções encontradas em literatura científica da enfermagem, sobre o quantitativo de horas de treinamento. Estudo realizado em 2001, encontrou 12,6 horas de treinamento/funcionário/ano para os trabalhadores de enfermagem. ${ }^{8}$ Em outra instituição a relação foi de 13,8 horas/funcionário/ano. ${ }^{5}$ Em 2009, as Unidades Afiliadas da Associação Paulista para o Desenvolvimento da Medicina estabeleceu 15 horas de treinamento/funcionário/ano. ${ }^{3}$

No primeiro semestre de 2012, um relatório de resultados hospitalares, provindo de um programa que busca avaliar a qualidade dos serviços, demonstrou que o índice de treinamento variou de 0,04 a 16,16 horas de treinamento global de cada hospital avaliado, sendo que $62,81 \%$ destas horas de treinamento foram destinadas aos profissionais de enfermagem. ${ }^{9}$

Anualmente, desenvolve-se um trabalho em organizações dos mais diversos segmentos, sendo que $73,3 \%$ das organizações pertencem a iniciativa privada, que identifica as principais tendências e indicadores do treinamento no Brasil. A pesquisa de 2012 encontrou a média de 45 horas anuais de treinamento para empresas privadas, sendo encontrado valor maior no setor público de 49 horas/ano. ${ }^{10}$

Estudos recentes produzidos por profissionais da enfermagem que utilizaram os resultados da assistência de enfermagem como parâmetro de avaliação, antes e após a aplicação de treinamento específico, demonstraram melhora significativa nos resultados da assistência e no desempenho geral da equipe após as atividades de treinamento. ${ }^{11-12}$

A enfermagem ressalta a importância das atividades de treinamento em ambientes onde o avanço e densidade tecnológica são muito acentuados, o que demanda conhecimentos continuamente atualizados. ${ }^{13}$ Corroborando com isto, resultados importantes de investigações científicas em âmbito nacional e internacional demonstram que a realização de treinamentos apresenta benefícios importantes para melhorar a segurança do paciente e, assim, a qualidade do atendimento de pacientes críticos. ${ }^{14-17}$ Como exemplos, são mencionados a redução na ocorrência de pneumonia associada à assistência ventilatória ${ }^{14}$; adequação na monitorização de pressão de cuff de próteses ventilatórias para evitar lesão traqueal ${ }^{15}$ e; aumento do conhecimento da equipe de enfermagem para monitorização neurointensiva. ${ }^{16-17}$

Diante do explicitado, possivelmente os resultados favoráveis à qualidade da assistência, mediante o treinamento, poderiam ser estendidos à organização como um todo, quando esta adotar o controle de horas ou índices de treinamento, tendo em vista que este indicador possui potencial para subsidiar o planejamento das ações de 


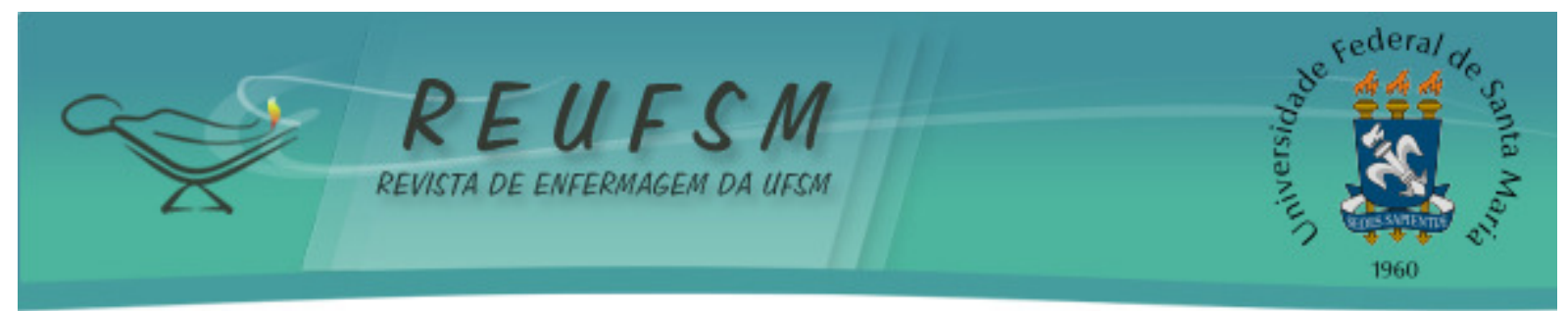

treinamento, bem como gerenciar os gastos dispendidos por estas, acarretando em maior assertividade das atividades.

Uma pesquisa ${ }^{18}$ buscando a opinião de enfermeiros sobre o conhecimento e a importância de indicadores de qualidade no gerenciamento de recursos humanos em enfermagem demonstrou que estes profissionais consideram o treinamento como elemento constitutivo para um indicador de qualidade dos recursos humanos de enfermagem. A periodicidade do treinamento e a definição de horas do mesmo são características ainda pouco estudadas pela saúde e a enfermagem, mas elementares quando na busca da avaliação da qualidade mediante indicadores. ${ }^{18}$

Desse modo, conflui-se para a afirmação da efetividade da capacitação contínua dos profissionais de enfermagem em prol à qualidade da assistência, bem como se sinaliza que a avaliação da assistência e dos recursos humanos de enfermagem mediante indicadores de qualidade, em especial no que se refere às atividades de treinamento desta equipe, constitui-se num instrumento de grande valor para a gestão hospitalar, da equipe de enfermagem e da qualidade da assistência.

\section{CONCLUSÃO}

0 estudo possibilitou a identificação de um indicador de qualidade de gerenciamento de recursos humanos de enfermagem, o índice de treinamento, numa instituição hospitalar pública e de ensino, determinado em 16,20 para enfermeiros e 3,98 para profissionais de enfermagem de nível médio.

Os resultados encontrados mostraram-se equivalentes e até superiores para profissionais enfermeiros e inferiores para técnicos e auxiliares de enfermagem, quando comparados à produção científica específica da enfermagem. Contudo, comparando os resultados com as empresas de vários setores, os resultados mostraram-se muito inferiores.

Diante disso, entende-se como uma limitação do estudo, a inexistência de padrões de qualidade previamente definidos no que tange ao indicador de qualidade estudado, 0 que dificultou a interpretação mais assertiva dos resultados. Contudo, o estudo traz contribuições para a enfermagem e à gestão hospitalar, no sentido de levantar resultados que possam ser comparados em estudos posteriores, bem como possa vir a fomentar a prática de uso de indicadores de qualidade no cotidiano gerencial de hospitais.

Salienta-se ainda, a importância do levantamento de necessidades de treinamento dos profissionais pelas organizações hospitalares e serviços de educação continuada, prática esta importante na aquisição de resultados favoráveis ao processo assistencial, as metas da organização e ao gerenciamento de recursos financeiros.

No que tange à enfermagem, os indicadores de treinamento demonstram que os índices possuem potencialidades e especificidades para a avaliação quantitativa de dados e possibilita aos profissionais responsáveis pelo gerenciamento de recursos humanos de enfermagem monitorar a situação das atividades de treinamento. Também, podem influir positivamente na qualidade da assistência de enfermagem através do planejamento adequado das atividades e execução de treinamentos que estejam relacionados às reais necessidades dos profissionais.

\section{REFERÊNCIAS}

1.Lima AFC. Treinamento e qualificação de profissionais. In: Padilha KG, Vattimo MFF, Silva C, Kimura M, organizadores. Enfermagem em UTI: cuidando do paciente crítico. Barueri: Manole; 2010.p.1269-88. 


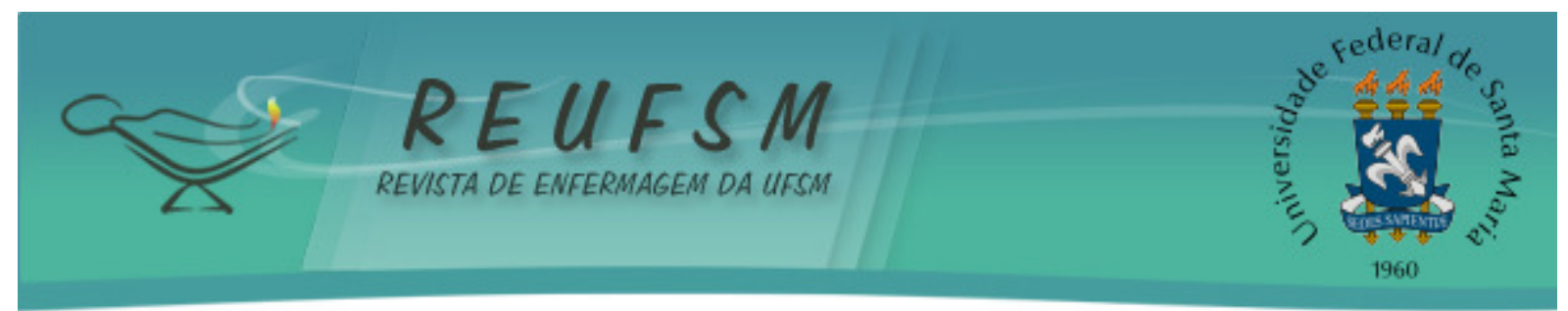

2.Chiavenato I. Iniciação à administração de recursos humanos. $4^{\mathrm{a}}$ ed. Barueri: Manole; 2010.

3.Nishio EA, Baptista MACS. Educação permanente: a evolução da educação continuada. Rio de Janeiro: Elsevier, 2009.

4. Rosseto M, Silva LAA. Ações de educação permanente desenvolvidas para os agentes comunitários de saúde. Cogitareenferm. 2010; 15(4):723-29.

5.Peres HHC, Leite MMJ, Gonçalves VLM. Educação continuada: recrutamento e seleção, treinamento e desenvolvimento e avaliação de desempenho profissional. In: Kurcgant $P$, coordenadora. Gerenciamento de enfermagem. $2^{\mathrm{a}}$ ed. Rio de Janeiro: Guanabara Koogan; 2010.

6.Gabriel CS, Melo MRAC, Rocha FLR, Bernardes A, Miguelaci T, Silva MLP. Utilização de indicadores de desempenho em serviço de enfermagem de hospital público.RevLatinoamEnferm. 2011;19(5):1247-1254.

7.Manual de indicadores de enfermagem NAGEH / Compromisso com a Qualidade Hospitalar (CQH). - 2.ed. São Paulo : APM/CREMESP; 2012.

8.Jericó MC. Análise dos custos dos programas de treinamento e desenvolvimento de pessoal de uma organização hospitalar [dissertação]. São Paulo (SP): Universidade de São Paulo; 2001. 200 p.

9.Programa de Controle de Qualidade Hospitalar. Relatório de indicadores do primeiro trimestre de 2012; [acesso em 2013 mar 05] Disponível em: http: / / www.cqh.org.br/portal/pag/area.php?p_narea=98

10.Associação Brasileira de Treinamento e Desenvolvimento. Pesquisa Anual MOT/ABTD./Revista T\&D.O retrato do treinamento no Brasil. [acesso em 2013 mar 05] Disponível em: http://portal.abtd.com.br/portal/home.html

11.Vituri DW, Cacciari P, Gvozd R, Kuwabara CGT, Cardoso MGP. Indicadores de qualidade como estratégia para a melhoria da qualidade do cuidado em um hospital universitário. CiencCuidSaude. 2010;9(4):782-90.

12. Jacondino CB, Severo DF, Rodrigues KR, Lima L, Einhardt RR, Amestoy SC. Educação em serviço: qualificação da equipe de enfermagem para o tratamento de feridas. Cogitareenferm. 2010;15(2):314-18.

13.Lazzari DD, Shmit N, Jung W. Educação continuada em unidades de terapia intensiva na percepção de enfermeiras. RevEnferm UFSM. [Internet] 2012; 2(1) [acesso em 2013mar 29]. Disponível

em: http://cascavel.ufsm.br/revistas/ojs/index.php/reufsm/article/view/4592.

14.Jansson $M$, Kääriäinen $M$, Kyngäs $H$. Effectiveness of educational programmes in preventing ventilator-associated pneumonia: a systematicreview. JornalofHospitallnfection. 2013;84(3):206-14

15.Penitenti RM, Vilches JIG, Oliveira JSC, Mizohata MGG, Correa DI, Alonso TRMB. Cuff pressure control in intensive care unit: training effects. RevBrasTerIntensiva.2010; 22(2):192-5.

16. Rosa NM, Lima JF, Inoue KC. Conhecimento da equipe de enfermagem sobre neurointensivismo e a influência da educação contínua. CiencCuidSaude. [Internet]. 


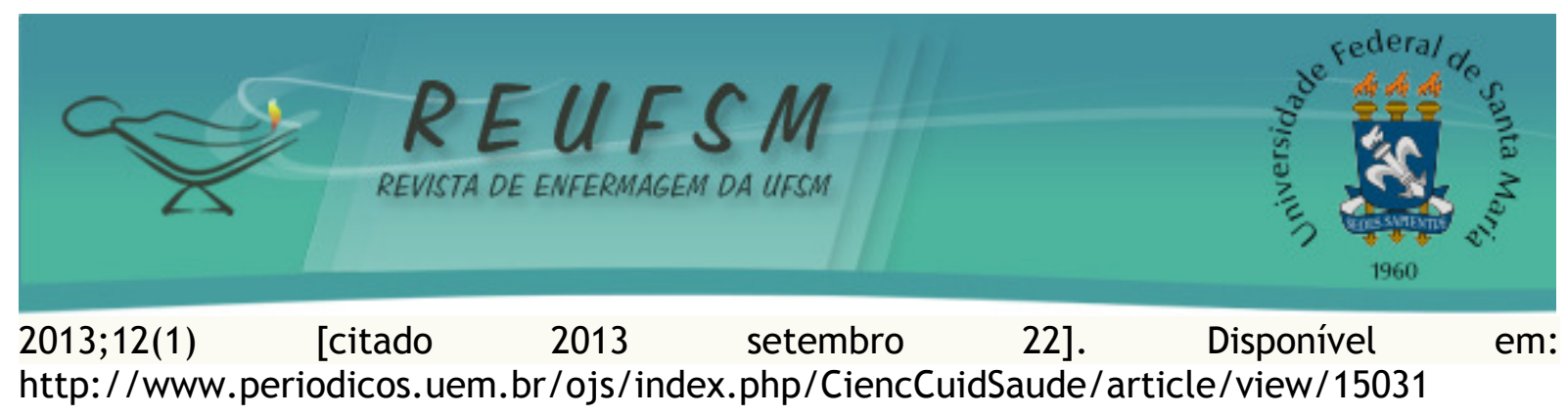

17. Seiler L, Fields J, Peach E, Zwerin S, Savage C. The effectiveness of a staff education program on the use of continuous ECG with patients in neuroscience intensive care units.JNeurosciNurs. 2012;44(2):1-5.

18. Vieira APM, Kurcgant P. Indicadores de qualidade no gerenciamento de recursos humanos em enfermagem: elementos constitutivos segundo a percepção de enfermeiros. Acta paulenferm. 2010; [acesso em 2013 abr 21]; 23(1): 11-15. Disponível em: http://www.scielo.br/scielo.php?script=sci_arttext\&pid=S0103-21002010000100002\&lng=pt.

http://dx.doi.org/10.1590/S0103-21002010000100002.

Data de recebimento: $24 / 04 / 2013$

Data de aceite: 22/01/2014

Contato com autor responsável: João Lucas Campos de Oliveira

Rua Voluntários da Pátria, 750. Centro. Pérola - PR. CEP: 87540-000.

E-mail: enfjoaolcampos@yahoo.com.br 